\title{
Implementation of Wideband Digital Beam Forming in the E-band
}

Val Dyadyuk, Xiaojing Huang, Leigh Stokes, Joseph Pathikulangara

ICT Centre, CSIRO, PO Box 76, Epping, NSW 2121, Sydney, Australia

This paper reports the test results of a small-scale prototype that implements a digitally beam-formed phased antenna array in the E-band. A four-channel dual-conversion receive RF module for 71-76 GHz frequency band has been developed and integrated with a linear end-fire antenna array. Wideband frequency-domain angle-of-arrival estimation and beam forming algorithms were developed and implemented using 1 Gbps OFDM QPSK symbols. Measured performance is very close to the simulated results and experimental data for an analogue-beam-formed array. This work is a stepping stone towards practical realization of larger hybrid arrays in the E-band.

Keywords: Active Array Antennas and Components, Hybrid and Multi-Chip Modules, Millimeter Wave Radio Communication

Corresponding author: V. Dyadyuk; email: val.dyadyuk@csiro.au; phone: +61 293724225

\section{INTRODUCTION}

High data rate millimeter-wave communication systems are of growing importance to the wireless industry. Future mobile and ad-hoc communications networks will require higher bandwidth and longer range. An ad-hoc or mobile (e.g. Inter-aircraft) network that relies on high gain antennas also requires beam scanning. With the advance in digital signal processing techniques, the adaptive antenna array is becoming an essential part of wireless communications systems. The use of adaptive antenna arrays for long-range millimeter-wave ad-hoc communication networks is particularly critical due to increased free space loss and reduced level of practically achievable output power [1]. Although pure digital beam forming allows for producing output signals with the maximum SINR, ease of on-line calibration and generation of many antenna patterns simultaneously, it is impractical for large wideband arrays due to two major reasons. Firstly, it is too costly since the cost of digital data processing is proportional to bandwidth and increases, at least, linearly with the number of elements. Secondly, the small separation of array elements in the E-band (71-86 GHz) leaves little room at the back of the array for connection of each RF chain associated with individual array elements to a digital beam former. A typical analogue RF chain is tightly packed behind 
each antenna element and includes a low noise amplifier (or power amplifier), frequency converter, local oscillator (LO), as well as the intermediate frequency (IF) or baseband circuitry. Each of these chains would require a number of DC, IF and control circuit interfaces. Therefore, a hybrid approach [1-3] where digital beam forming technique is applied to a smaller number of units (analogue beam formed sub-arrays) is preferable. This provides a significant saving in both the amount of digital signal processing and the number of physical connections between the RF front end and digital beam former. A small scale Eband phased antenna array has been developed [1] for an experimental verification of the hybrid beam forming concepts [2,3]. Analogue beam forming of this array using 6-bit phase shifters and attenuators at IF has been reported earlier in $[4,7]$. In this paper, we report the test results of an E-band prototype that implements a digital beam-forming phased antenna array.

\section{E-BAND PHASED ANTENNA ARRAY PROTOTYPE}

The prototype has been developed to demonstrate a communications system with gigabit per second data rates using an electronically steerable array as an initial step towards fully ad-hoc communications systems. The prototype configuration is flexible and can be used for experimental verification of both analogue and digital beam forming algorithms. Fig. 1 shows the equipment configuration for digital beam forming experiments. The steerable receive array is mounted on a rotator providing mechanical steering in the azimuth plane for the array pattern measurement.

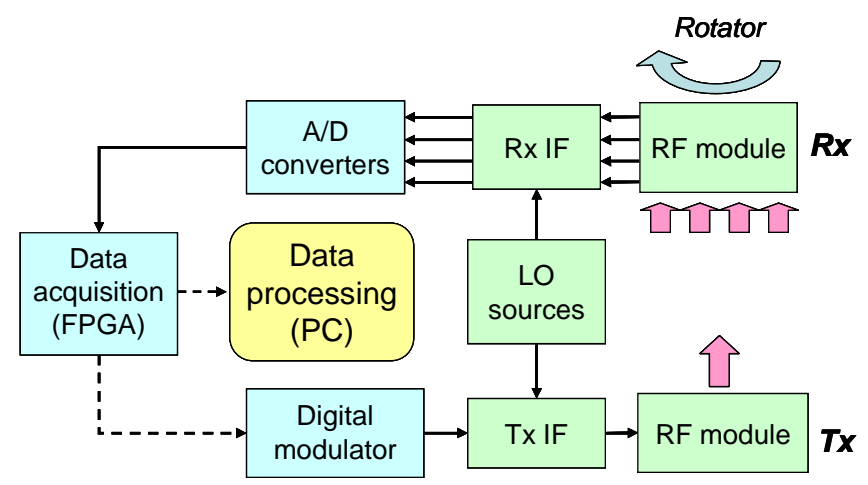

a)

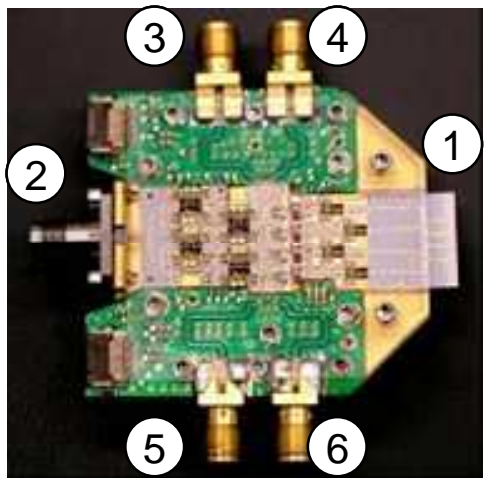

b)

Fig. 1. a) Block diagram of the test setup; b) Photograph of the RF module assembly where: 1 is the antenna array; 2 is the LO input; 3-6 are IF outputs.

IF to baseband frequency conversion was implemented in the receive (Rx IF) and transmit (Tx IF) IF modules. The Rx IF module has been developed in two versions. For digital beam forming (DBF), each of the IF analogue signals was digitised and streamed to a PC for data processing and beam forming as shown in Fig. 2.. For analogue beam forming (ABF) [4, 7], the phase and magnitude of each channel was controlled by 6-bit phase shifters and attenuators and a combined IF signal was de-modulated using a modem reported in [5]. This 
replaces the digitizer shown in Fig. 1.a for the DBF configuration. The demonstrator also includes a digital modulator reported in [5] and a single-channel transmitter [1].

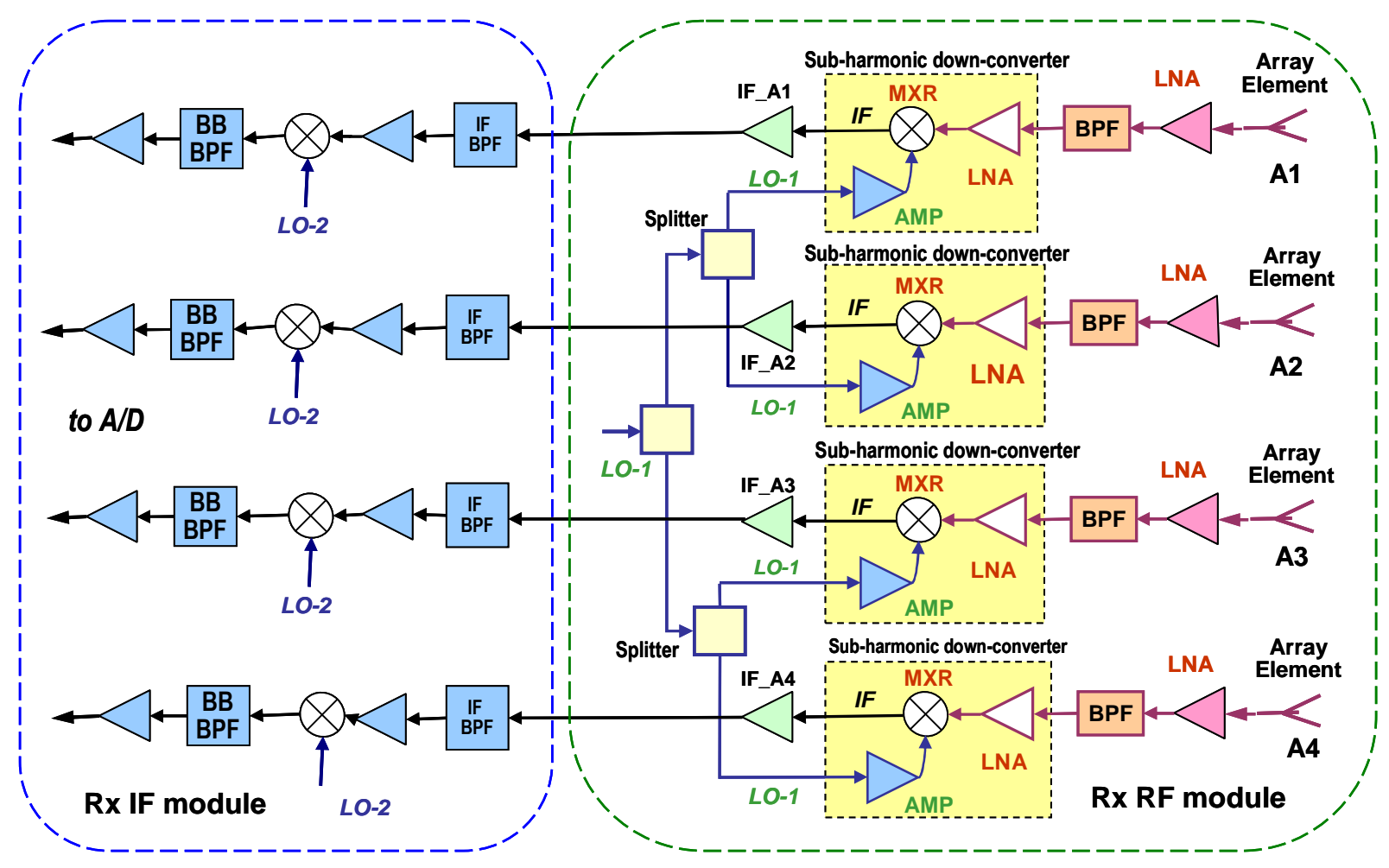

Fig. 2. Block diagram of the receiver configured for DBF experiments.

The transmit module has been built using the up-converter [8], a commercial low-pass filter, a medium power amplifier and a corrugated horn antenna. Bench test results have been reported in [1]. The main functional block of the prototype is a four-channel receive RF module $[1,4]$ integrated with a linear end-fire Quasi-Yagi antenna array [6, 7]. Array element spacing was 2 $\mathrm{mm}$ (or 0.48 wavelengths at the carrier frequency) to suppress appearance of grating lobes for scanning angles up to \pm 42 degrees. The RF module uses sub-harmonic frequency converters at the LO frequency of $38 \mathrm{GHz}$. For each channel we have used a combination of CSIRO and commercial off-the-shelf MMICs similar to those reported earlier for a single-channel receiver $[8,9]$. The IF pre-amplifiers, interconnecting, matching, and group delay equalization circuits have been developed using a standard commercial thin-film process on ceramic substrate. Fig. 1.b shows a photograph of the assembled RF module. It includes 16 MMICs, 12 types of microwave boards (on 127um Alumina substrate), 140 microwave passives, and about 400 wire-bond connections. The receiver is usable over the frequency range of 71 to 76 $\mathrm{GHz}$ at the sub-harmonic $\mathrm{LO}$ of 38 to $39 \mathrm{GHz}$ and intermediate frequency 1 to $7 \mathrm{GHz}$.

Typical conversion gain was $6 \pm 1 \mathrm{~dB}$ over the operating RF and IF frequency range of 71.5 $72.5 \mathrm{GHz}$ and $3.5-4.5 \mathrm{GHz}$ respectively. The maximum magnitude imbalance between each of four channels was below $\pm 1.5 \mathrm{~dB}$. Bench test results for the receive module have been reported in [1]. The IF to baseband $(1-2 \mathrm{GHz})$ frequency conversion was implemented in the Rx IF module [7]. 
The digitizer consists of an Analogue-to-Digital Converter (ADC) and a Field Programmable Gate Array (FPGA). The ADC is an EV8AQ160 (E2V Semiconductors) 8-bit device. This converter is used in dual channel mode, each channel sampling at 2 Giga samples per second. The ADC has an effective number of bits (ENOB) of 6 bits at the frequency of operation and input bandwidth of $2 \mathrm{GHz}$. The FPGA for signal processing is a Xilinx V5SX95T. The experimental setup uses two such digitizers as shown in Fig. 3 giving a total of four ADCs and two FPGAs. The interconnections labelled "3" in the photograph Fig. 2 have enough bandwidth to transport digital signals after pre-processing on one FPGA to the other for digital beam forming. In this experiment, the samples are captured on the FPGA internal memory and uploaded to a PC for processing.

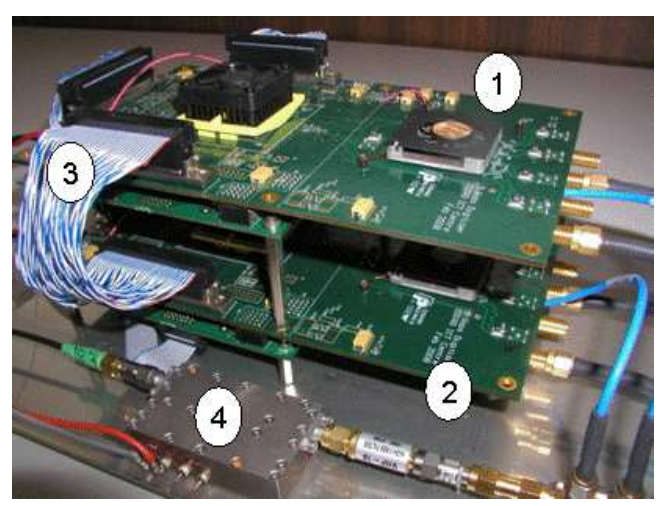

Fig. 3. Photograph of the digitizer module assembly where 1 and 2 are two identical digitizers, 3 is interconnecting cable and 4 is a sampling oscillator.

All measurements were conducted in the CSIRO 12m far field anechoic chamber as shown in Fig. 4. Transmitter, digital modulator and control equipment were located on the outside of the chamber. The available signal to noise ratio was above $33 \mathrm{~dB}$ for the measurement distance up to $6 \mathrm{~m}$, but most of the tests were conducted at the distance of $2.2 \mathrm{~m}$ to minimize unwanted reflections from the walls and ceiling of the chamber.
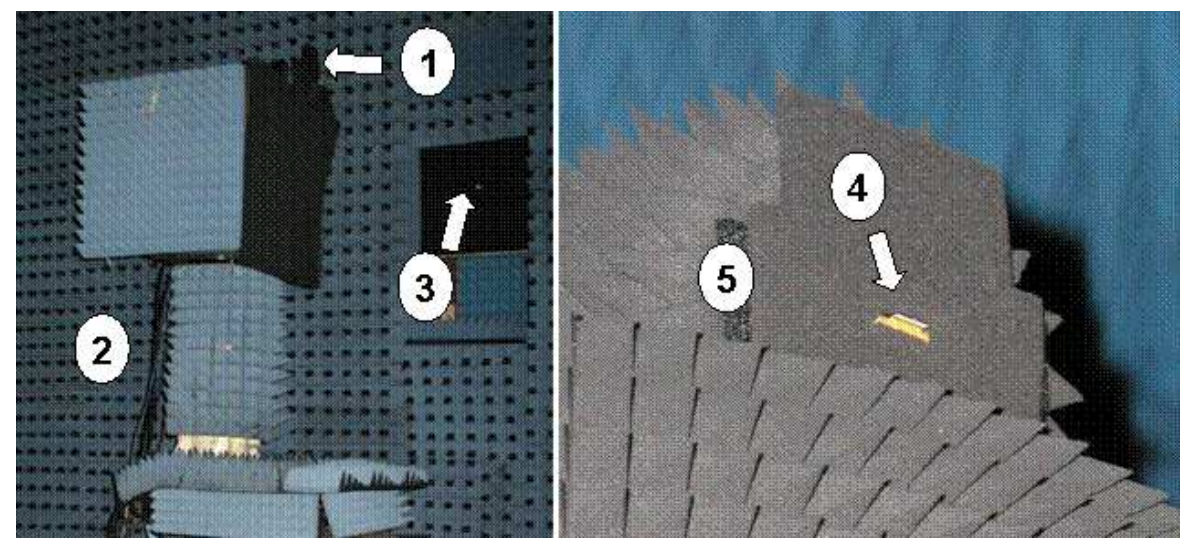

Fig. 4. System test setup in the $12 \mathrm{~m}$ far field anechoic chamber where 1 is the receive array RF module, 2 is the rotator and 3 is the transmit antenna aperture, 4 is the receive antenna array masked with absorbers 5 . 


\section{DIGITAL BEAM FORMING ALGORITHM}

\section{A) Received Signal Model}

Consider a linear wideband array with $M$ elements and element spacing $d$. Denoting the received baseband signal from the $m$ th element as $x_{m}(t)$, the frequency domain model of the received signal can be expressed as

$$
X_{m}(f)=S(f) H_{m}(f) P(f, \theta) e^{j \frac{f+f_{c}}{f_{c}} \lambda_{c} d m \sin \theta}+Z_{m}(f), \text { for }|f| \leq \frac{B}{2}, m=0,1, \ldots, M-1,
$$

where $X_{m}(f)$ is the Fourier transform of $x_{m}(t), S(f)$ is the information-bearing reference signal in the frequency domain, $H_{m}(f)$ is the channel frequency response of the RF chain for the $m$ th element, $P(f, \theta)$ is the element radiation pattern, $f_{c}$ and $\lambda_{c}$ are the frequency and wavelength of the RF carrier respectively, $Z_{m}(f)$ is zero-mean complex Gaussian noise, and $B$ is the bandwidth of the signal.

For digital implementation, the received time domain signals $x_{m}(t), \quad m=0,1, \ldots, M-1$, are sampled at $t=n T$, i.e., $x_{m}[n]=x_{m}(n T)$ is the received signal sequence from the $m$ th element, where $T=\frac{1}{B}$ is the sampling period, and divided into blocks of size $N$. Each block is converted into the frequency domain by fast Fourier transform (FFT). The discrete model is then expressed as

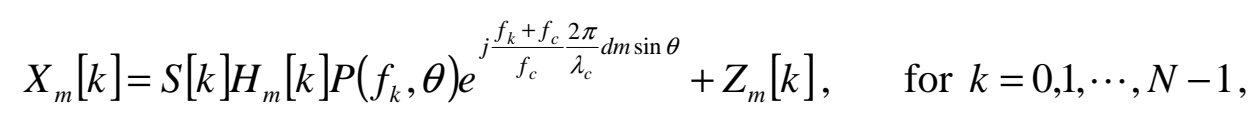

where the index $k$ represents a discrete frequency

$$
f_{k}=\left\{\begin{array}{lr}
\frac{k B}{N}, & \text { for } k=0,1, \cdots, \frac{N}{2}-1 \\
\frac{(k-N) B}{N}, & \text { for } k=\frac{N}{2}, \frac{N}{2}+1, \cdots, N-1
\end{array}\right.
$$

$X_{m}[k], S[k], H_{m}[k]$, and $Z_{m}[k]$ are the $m$ th received frequency-domain signal, reference signal, frequency response, and noise at discrete frequency $f_{k}$.

\section{B) Frequency Domain Beamforming}

In order to achieve maximised signal to noise ratio, the frequency domain digital beamforming is performed as follows. First, the signal sequence received at each element is synchronised, and the combined channel frequency response together with the phase shift introduced by the incident angle (i.e., $H_{m}[k] P\left(f_{k}, \theta\right) e^{j \frac{f_{k}+f_{c}}{f_{c}} \frac{2 \pi}{\lambda_{c}} d m \sin \theta}$ as a whole) is estimated using the training sequence during the preamble period. Then, the synchronised signal after 
the preamble is converted into the frequency domain by FFT, and the channel frequency response at each discrete frequency is compensated by the inverse of the channel frequency response. Finally, the compensated frequency domain signals for all elements are summed up and the combined signal is converted into time domain by inverse FFT (IFFT).

To obtain the angle of arrival (AoA) information or form a beam for a specified incident angle, the receiver array needs to be calibrated. This calibration can be done for each element after compensation of the channel frequency response $H_{m}[k]$ only. $H_{m}[k]$ can be estimated in advanced from the signal preamble received at 0 degree incident angle for each element.

The frequency domain beamforming at a specified incident angle is performed as follows. First, the signal sequence received at each element is synchronised using the preamble. Then, the synchronised signal after the preamble is converted into the frequency domain by FFT followed by calibration. After calibration, the frequency domain signals are weighted with coefficients

$$
W_{m}\left(f_{k}\right)=e^{-j \frac{f_{k}+f_{c}}{f_{c}} \frac{2 \pi}{\lambda_{c}} d m \sin \theta_{0}}
$$

where $\theta_{0}$ is the incident angle at which a beam is to be formed. The weighted signals are finally summed up and converted into the time domain by IFFT to obtain the beamformed output signal. The structure of this frequency domain digital beamformer is shown in Fig. 5 .

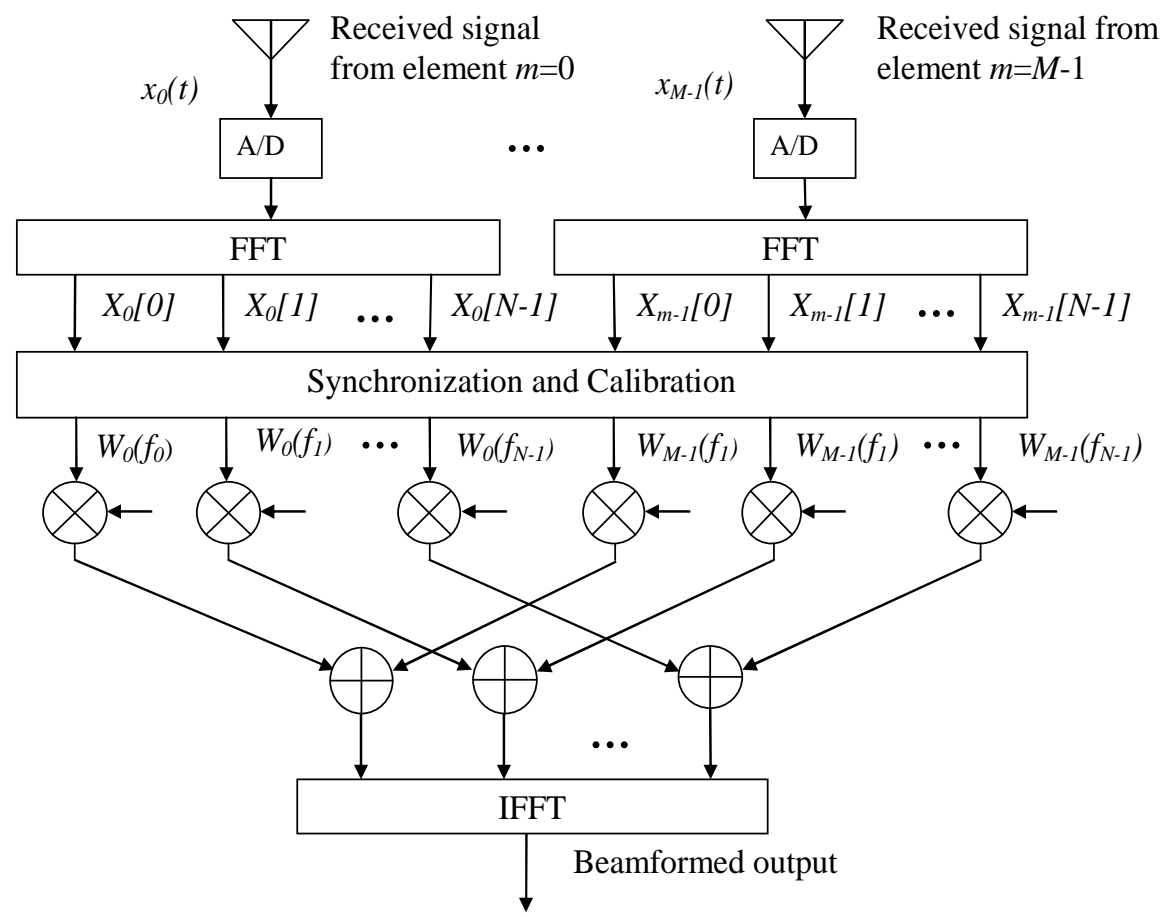

Fig. 5. Frequency-domain digital beam former structure. 


\section{TEST RESULTS}

The DBF experiments were conducted by transmitting a 1 Gbps OFDM test signal with $N=128$ subcarriers used in the $B=0.5 \mathrm{GHz}$ bandwidth. QPSK is used for data modulation. To facilitate synchronization and channel estimation, a preamble composed of 5 predefined OFDM symbols is pre-pended before the information bearing reference sequence. The signal format is shown in Fig. 6, where $s_{0}$ denotes the predefined training sequence and $s_{1}, \ldots, s_{n}$ denote the reference sequence composed of $n$ OFDM symbols with pseudo-random data modulated by QPSK.

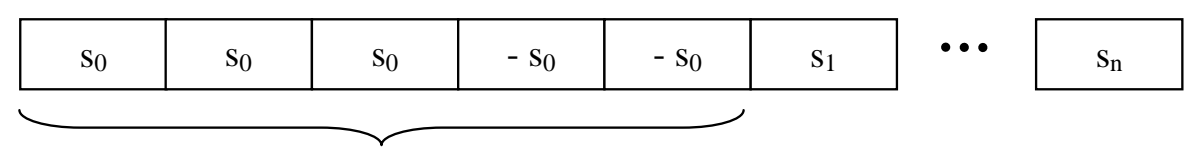

Preamble

Fig. 6. Transmitted signal format.

To reduce hardware cost as well as overcome the practical impairments involved in frequency conversion, such as I/Q imbalance, the received RF signal is first converted into IF signal with centre frequency $1.5 \mathrm{GHz}$ and thus only one A/D converter operating at $2 \mathrm{GHz}$ is necessary. Each OFDM symbol has 512 samples in the time domain after A/D conversion at IF. The digital complex baseband signal is then obtained through digital down-conversion. The receive array was rotated in the azimuth plane from $-75^{\circ}$ to $75^{\circ}$ in $1^{\circ}$ step. For each azimuth angle the received signals were digitized, captured on the FPGA internal memory, uploaded to a PC and processed as described in Section III.B. Fig. 7 shows the transmitted digital IF signal with 5120 samples. The first 5 OFDM symbols form the preamble, followed by 5 random reference symbols. The received signals from the 4 channels in the array with incident angle 0 degree, each having 16384 samples, are captured at the receiver FPGA after A/D conversion, which are shown in Fig. 8.

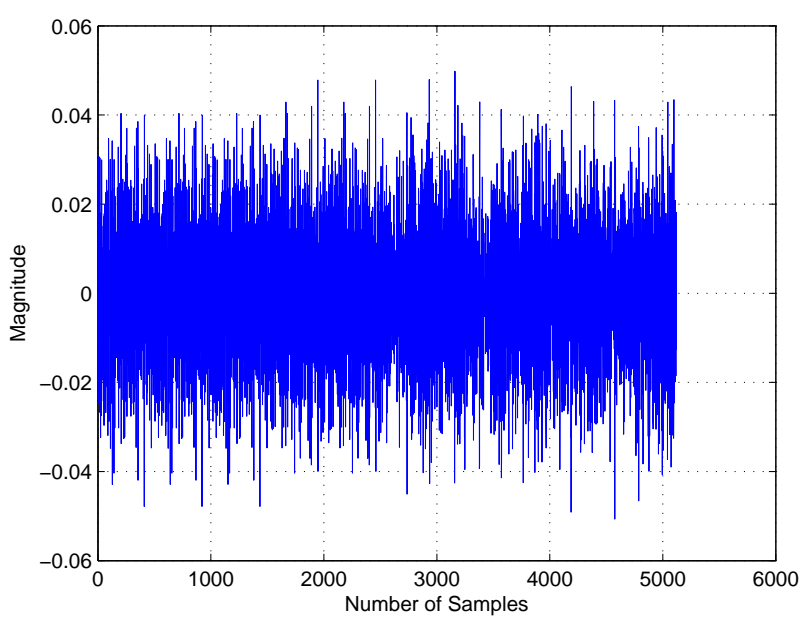

Fig. 7. Transmitted digital IF signal. 

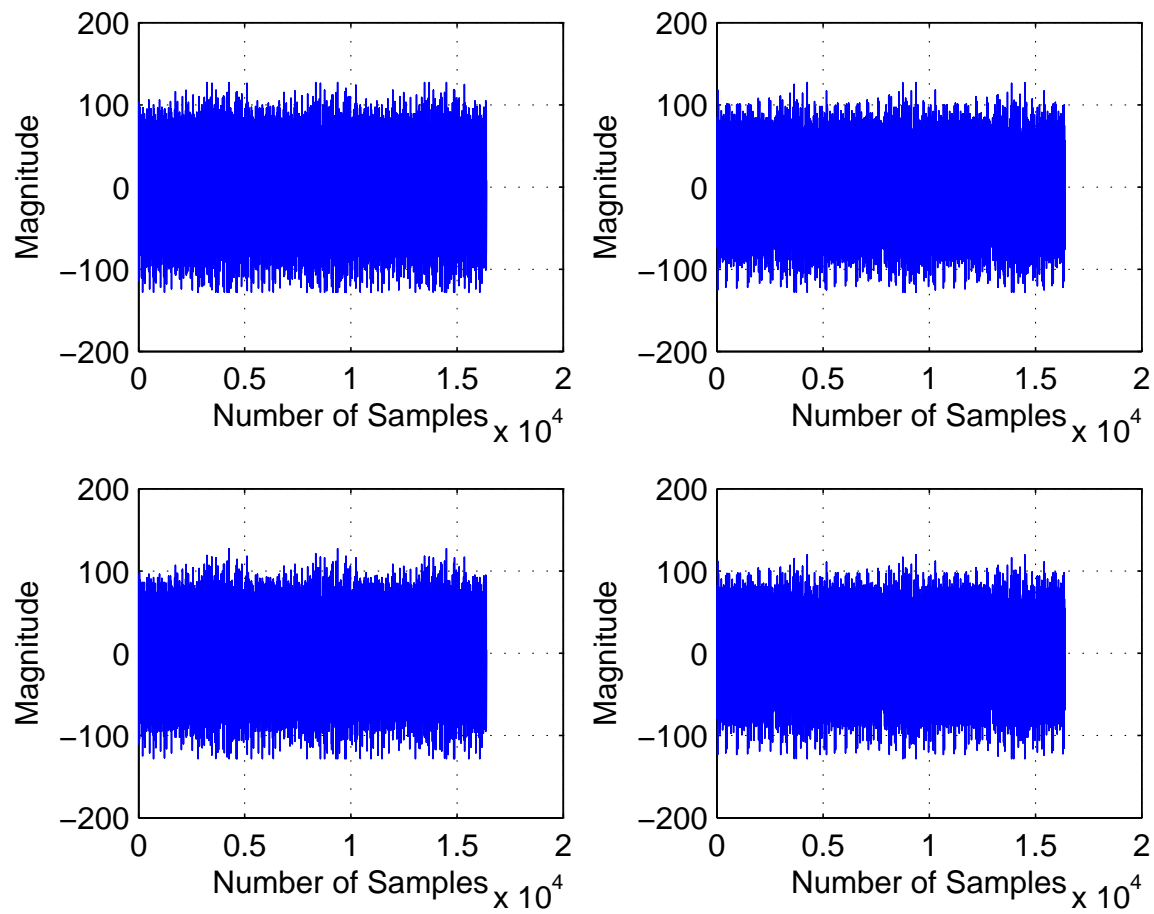

Fig. 8. Captured 4 channel received signals at $0^{\circ}$ azimuth.
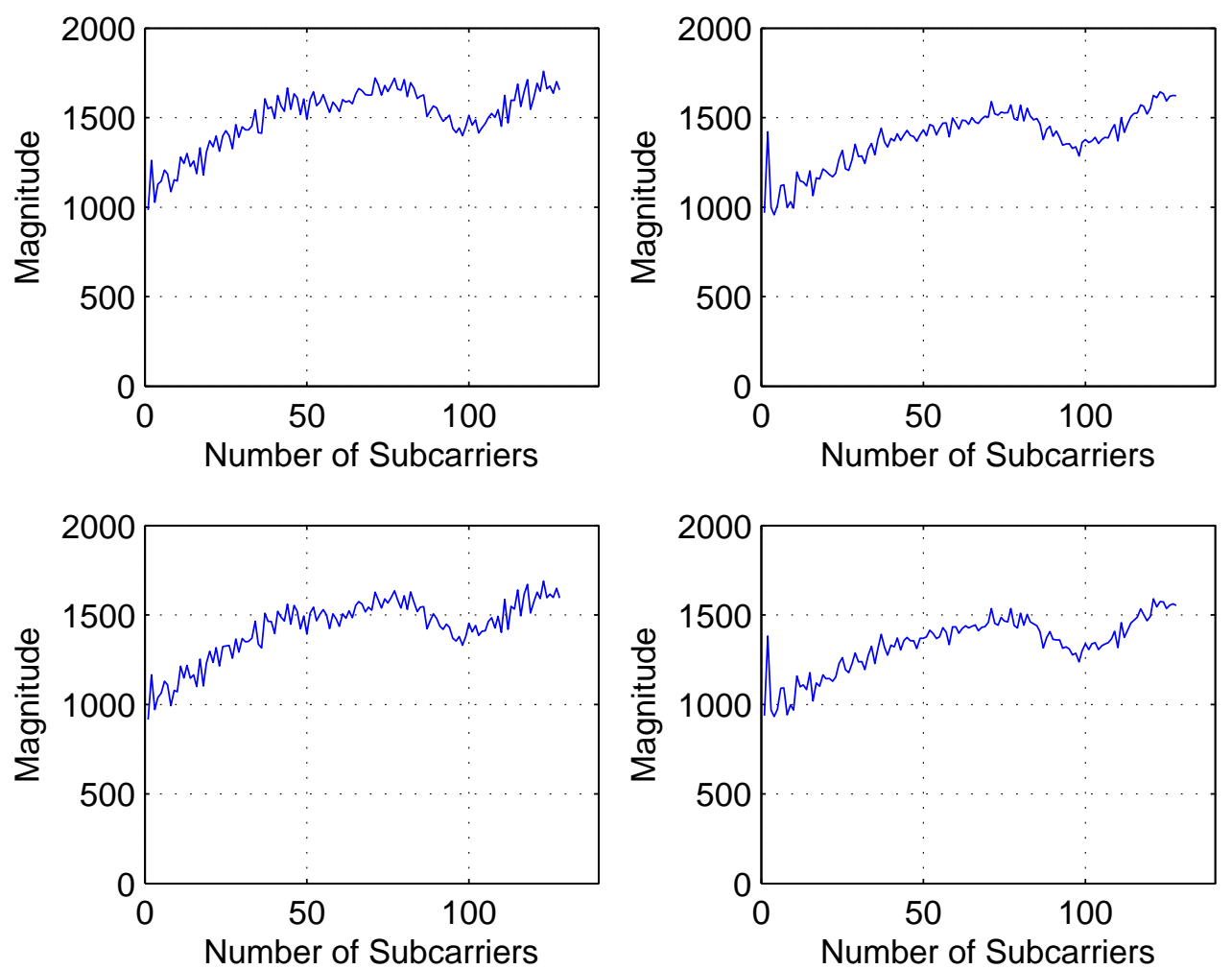

Fig. 9. Magnitude frequency response for each channel at $0^{\circ}$ azimuth. 

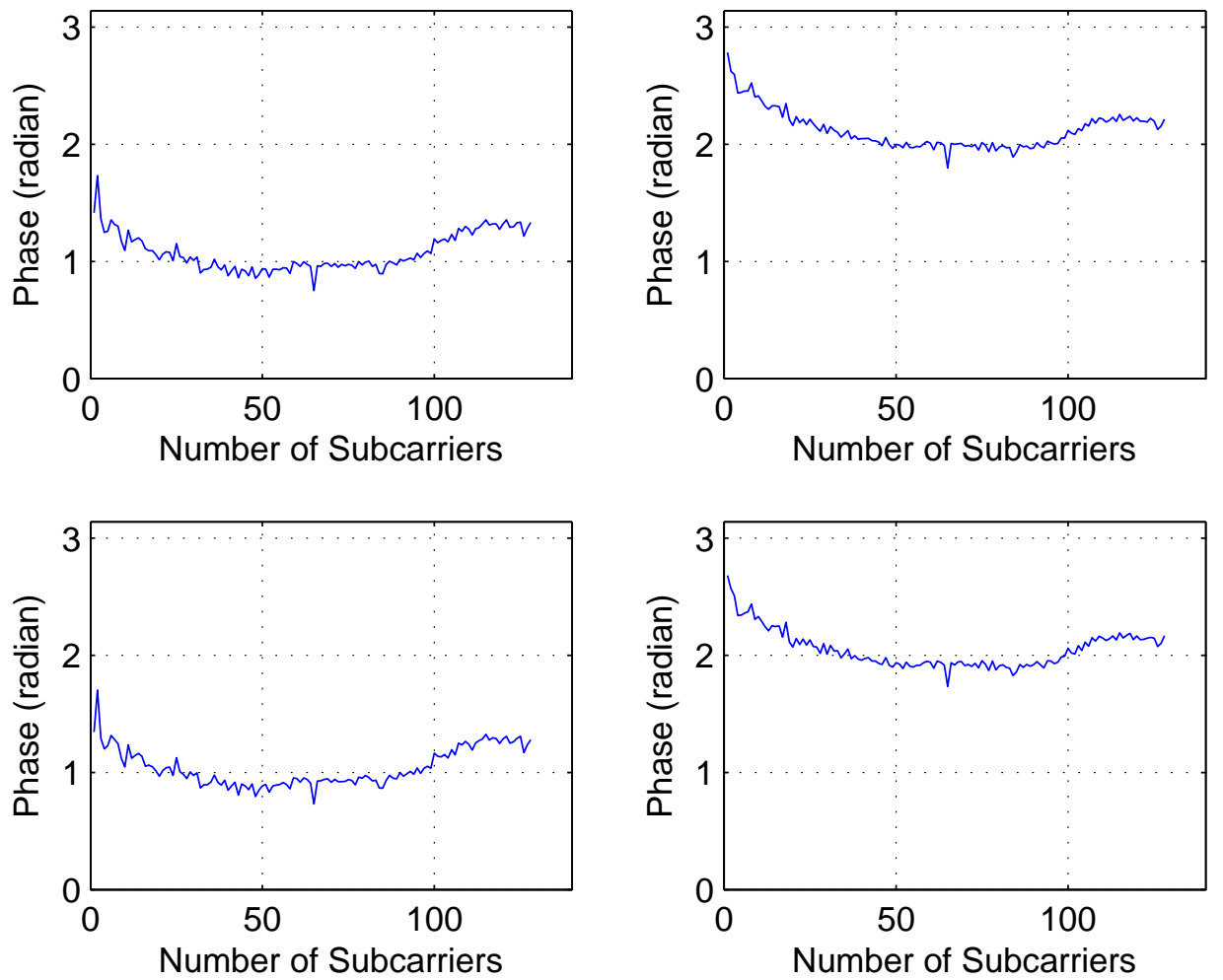

Fig. 10. Phase frequency response for each channel at $0^{\circ}$ azimuth.

After synchronization, channel estimation is performed for each channel using the fifth OFDM symbol in the preamble. The magnitude frequency response and phase frequency response for each channel are shown in Fig. 9 and Fig. 10 respectively. The average phases (represented as complex numbers) for the 4 channels are calculated as $0.4773+\mathrm{j} 0.8787$, $0.5120+\mathrm{j} 0.8590,0.5087+0.8610$, and $-0.4503+\mathrm{j} 0.8929$, which are used for calibration.

A separate ABF experiment was also conducted at selected incident angles using the same antenna array for comparison and verification of the DBF results. Fig. 11 compares the Eplane co-polar array radiation patterns at $0^{\circ}$ azimuth computed by the DBF and measured for the $A B F[7]$. In both experiments the calibration was performed at zero degree azimuth angle. For the ABF [7], the array has been calibrated by cancelling the main beam to obtain a null at $0^{\circ}$ azimuth. The null ABF calibration reference is also shown in Fig. 11.

The E-plane co-polar radiation patterns of the DBF array for a selection of other positive and negative azimuth angles are shown in Fig. 12 with the magnitude normalized to the maximum of the main beam at zero degree azimuth angle. 


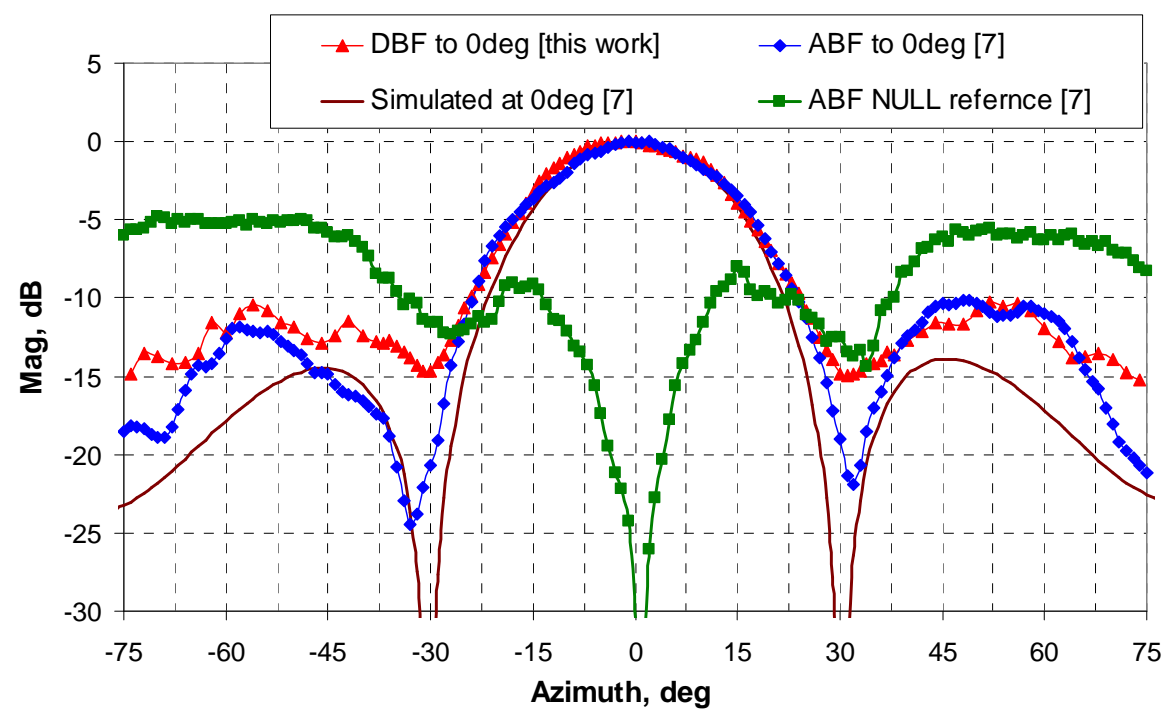

Fig. 11. Comparison of the E-plane co-polar radiation patterns for digitally (DBF) and analogue beam formed (ABF) array [7] at $0^{\circ}$ azimuth.

Measured E-plane co-polar radiation patterns for the ABF array [7] are appended to the computed DBF results shown in Fig. 12. Measured array gain was $9.5 \mathrm{dBi}$ for steering angles below $11^{\circ}$ and reduced to approximately $8.4 \mathrm{dBi}$ at the steering angle of $\pm 35^{\circ}$. Obtained DBF results were in a very close agreement with those measured for the ABF array and EM simulation predictions [7] for steering angles within $\pm 40^{\circ}$. The magnitude and angle of the $\mathrm{DBF}$ and $\mathrm{ABF}$ main beam patterns were within $1 \mathrm{~dB}$ and 1 degree respectively. Beam steering accuracy of 1 deg has been achieved by both DBF and ABF methods.
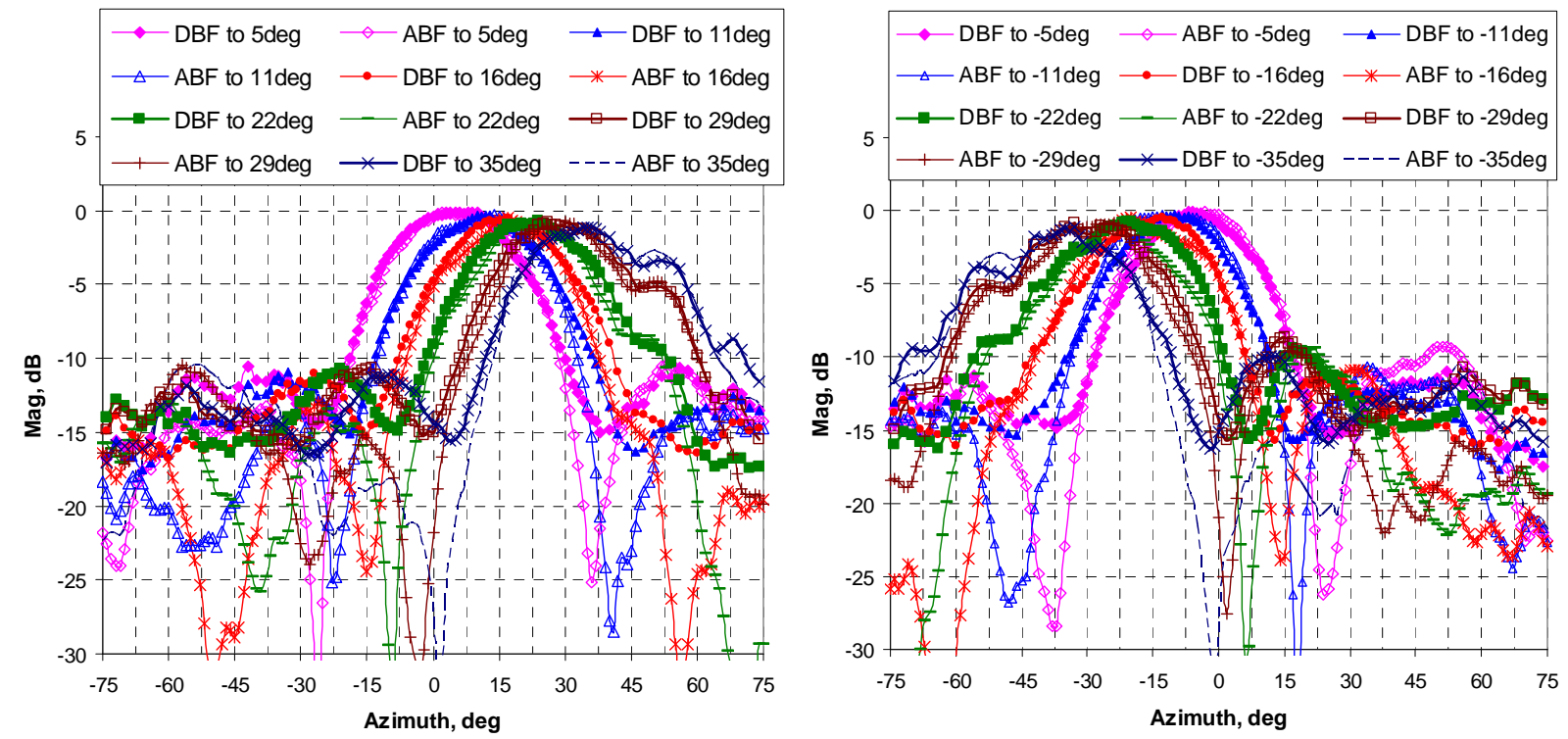

Fig. 12. E-plane co-polar radiation patterns for digitally (DBF) and analogue beam formed $(\mathrm{ABF})$ array [7] for a selection of positive and negative azimuth angles. 


\section{CONCLUSION}

A steerable E-band receive array demonstrator that implements a four-element linear antenna array has been tested using a wideband frequency-domain digital beam forming algorithm. Obtained DBF array patterns were very close to those measured for the analogue beam formed array and EM simulated predictions. Beam steering accuracy of 1 deg has been achieved for steering angles within $\pm 40^{\circ}$. Demonstrated wideband adaptive digital beam forming along with validated phase-only $\mathrm{ABF}$ at IF can be used for hybrid beam forming of larger arrays. To our knowledge, this work represents the first experimental results on the digitally beam formed antenna array in the E-band.

\section{REFERENCES}

[1] Dyadyuk, V.; Guo, Y.J.: Towards multi-gigabit ad-hoc wireless networks in the E-band, Global Symposium on Millimeter Waves (GSMM2009), Sendai, Japan, 2009.

[2] Huang, X.; Guo, Y.J.; Bunton, J.D.: A hybrid adaptive antenna array. IEEE Tran. on Wireless Communications, vol. 9, no. 5 (2010), 1770-1779.

[3] Huang, X.; Guo, Y.J.; Bunton, J.D.: Adaptive AoA Estimation and Beamforming with Hybrid Antenna Arrays, IEEE VTC-Fall, Alaska, USA, 2009.

[4] Dyadyuk, V.; Stokes, L.: Wideband Adaptive Beam Forming in the E-band: Towards a Hybrid Array, Global Symposium on Millimeter Waves, Incheon, Korea, 2010.

[5] Dyadyuk, V.; Bunton, J.D.; Pathikulangara J.; et al.: A Multi-Gigabit Mm-Wave Communication System with Improved Spectral Efficiency, IEEE Trans. on MTT, Vol. 55, Issue 12, Part 2 (2007), 2813-2821.

[6] Deal, W. R.; Kaneda, N.; Sor, J.; Qian, Q.Y.; Itoh, T.: A new quasi-Vagi antenna for planar active antenna arrays, IEEE Trans. on MTT, Vol. 48, No.6 (2000), 910-918.

[7] Dyadyuk, V.; Stokes, L.; Nikolic, N.; Weily, A.R.: Demonstration of Adaptive Analogue Beam Forming in the E-band, Journal of the Korean Institute of Electromagnetic Engineering \& Science, Vol. 10, No. 3 (2010), 138-145.

[8] Dyadyuk, V.; Stokes, L.; Shen, M.: Integrated W-band GaAs MMIC Modules for MultiGigabit Wireless Communication Systems, Global Symposium on Millimeter Waves, Nanjing, China, 2008.

[9] Dyadyuk, V.; Archer, J.W.; Stokes, L.: W-Band GaAs Schottky Diode MMIC Mixers for Multi-Gigabit Wireless Communications, In: Advances in Broadband Communication and Networks, River Publishers, Denmark, 73 - 103. 
Val Dyadyuk received B.Sc. and M.Sc. degrees in electrical engineering in 1968 and 1970 respectively from Kharkov Institute for Radio Electronics in Ukraine. He is currently the Research Team Leader in Microwave Systems at CSIRO ICT Centre, Sydney. Previously he held positions of the Head of the Microwave Engineering Branch at Institute for Radio Physics and Electronics, NASU, Ukraine, and Director of Research at the SCAD Scientific \& Industrial Group (Kharkov, Ukraine). Mr. Dyadyuk is a recipient of the Engineers Australia (Sydney Division) R\&D Excellence Award 2007, and a joint recipient of the CSIRO Chairman's Medal 2007, and the Australian Engineering Excellence Award 2007 for exceptional research in gigabit wireless communications. He has published three book chapters and over 40 journal and conference papers, and holds ten patents. His research interests include millimeter-wave wireless communication systems, EM simulations and design of GaAs and InP MMIC's and various microwave circuits.

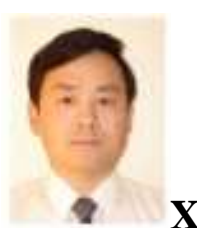

Xiaojing Huang received his Bachelor of Engineering, Master of Engineering, and Ph.D. in electronic engineering degrees from Shanghai Jiao Tong University, China, in 1983, 1986, and 1989 respectively. From 1989 to 1994, Dr. Huang worked in the Electronic Engineering Department of Shanghai Jiao Tong University as a Lecturer since 1989 and an Associate Professor since 1991. Form 1994 to 1997 he was the Chief Engineer with Shanghai Yang Tian Science and Technology Corporation Limited, China. In 1998 - 2004 he was a Senior and Principal Research Engineer at Motorola Australian Research Centre in Sydney. From 2004 to 2009, Dr. Huang was an Associate Professor in the School of Electrical, Computer and Telecommunications Engineering, University of Wollongong, Australia. In March, 2009, he joined the CSIRO ICT Centre, Australia, as a Principal Research Scientist. His research interests are in communications theory, digital signal processing, and wireless communications networks.

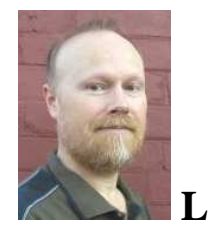

Leigh Stokes received B.A. degree in economics from Macquarie University, Sydney, Australia in 1978, Electronics Engineering Certificate from North Sydney TAFE in 1988, and Post-Graduate Certificate in project management from University of Technology, Sydney, Australia in 2003. He was the Principal Technical Officer in charge of maintenance (1992-1998) at Waverley Radio Terminal, for Telstra in Sydney, which housed digital radio links. He joined the CSIRO ICT Centre, Sydney, Australia in 2002. Mr. Stokes is the Manager for the $\mathrm{GHz}$ Testing Facility for millimeter wave measurements including on-wafer probing of MMICs. He is also responsible for integration of the research prototypes, test setup, and development of software for automated measurements. Mr. Stokes is a joint 
recipient of a Millimeter-Wave Best Paper Award from the 9th Topical Symposium on Millimeter Waves (TSMMW 2007) and a joint recipient of CSIRO Chairman's Medal in 2007 for exceptional research in gigabit wireless communications.

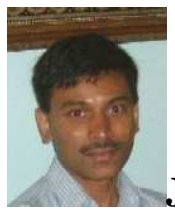

Joseph Pathikulangara received a B.E. degree in electronics and communication engineering from Indian Institute of Science in 1984 and M.Tech degree in computer science and engineering from IIT, Bombay in 1991. He was with the Defense Research and Development Organization in India developing command, control and communication systems for missile and EW projects between 1984 and 1995. Since joining CSIRO in Sydney, Australia in 1995, he has been developing signal processors and software radios in various forms for several application spaces. He is responsible in developing specialist expertise in leading edge digital techniques, signal processing and FPGA technologies and maintaining flexible and configurable building blocks that can be rapidly adapted to provide quick engineering solutions. Mr. Pathikulangara is a joint recipient of CSIRO Chairman's Medal in 2007 for exceptional research in gigabit wireless communications.

\section{List of figures}

Fig. 1. a) Block diagram of the test setup; b) Photograph of the RF module assembly where: 1 is the antenna array; 2 is the LO input; 3-6 are IF outputs.

Fig. 2. Block diagram of the receiver configured for DBF experiments.

Fig. 3. Photograph of the digitizer module assembly where 1 and 2 are two identical digitizers, 3 is interconnecting cable and 4 is a sampling oscillator.

Fig. 4. System test setup in the $12 \mathrm{~m}$ far field anechoic chamber where 1 is the receive array RF module, 2 is the rotator and 3 is the transmit antenna aperture, 4 is the receive antenna array masked with absorbers 5 .

Fig. 5. Frequency-domain digital beam former structure.

Fig. 6. Transmitted signal format.

Fig. 7. Transmitted digital IF signal.

Fig. 8. Captured 4 channel received signals at $0^{\circ}$ azimuth.

Fig. 9. Magnitude frequency response for each channel $0^{\circ}$ azimuth.

Fig. 10. Phase frequency response for each channel $0^{\circ}$ azimuth.

Fig. 11. Comparison of the E-plane co-polar radiation patterns for digitally (DBF) and analogue beam formed $(\mathrm{ABF})$ array [7] at $0^{\circ}$ azimuth.

Fig. 12. E-plane co-polar radiation patterns for digitally (DBF) and analogue beam formed $(\mathrm{ABF})$ array [7] for a selection of positive and negative azimuth angles. 